\title{
AIAA-96-3444
}

\section{Free-Flight Investigation of Forebody Blowing for Stability and Control}

\author{
Jay M. Brandon \\ NASA Langley Research Center \\ Hampton, VA 23681-0001 \\ James M. Simon \\ WL/FIGC \\ WPAFB, OH 45433-7531 \\ D. Bruce Owens \\ National Research Council \\ NASA Langley Research Center \\ Hampton, VA 23681-0001 \\ Jason S. Kiddy \\ University of Maryland \\ College Park, MD 20742-2001
}

\section{Atmospheric Flight Mechanics Conference}

July 29-31, 1996/San Diego, CA

For permission to copy or republish, contact the American Institute of Aeronautics and Astronautics 370 L'Enfant Promenade, S.W., Washington, D.C. 20024 



\title{
FREE-FLIGHT INVESTIGATION OF FOREBODY BLOWING FOR STABILITY AND CONTROL
}

\author{
Jay M. Brandon* \\ NASA Langley Research Center \\ Hampton, VA 23681-0001 \\ James M. Simon† \\ WL/FIGC \\ WPAFB, OH $45433-7531$ \\ D. Bruce Owens \\ National Research Council \\ NASA Langley Research Center \\ Hampton, VA 23681-0001 \\ and \\ Jason S. Kiddy§ \\ University of Maryland \\ College Park, MD 20742-0001
}

\begin{abstract}
A free-flight wind-tunnel investigation was conducted on a generic fighter model with forebody pneumatic vortex control for highangle-of-attack directional control. This is believed to be the first flight demonstration of a forebody blowing concept integrated into a closed-loop flight control system for stability augmentation and control. The investigation showed that the static wind tunnel estimates of the yaw control available generally agreed with flight results. The control scheme for the blowing nozzles consisted of an on/off control with a deadband. Controlled flight was obtained for the model using forebody blowing for directional control to beyond $45^{\circ}$ angle of attack.
\end{abstract}

\section{NOMENCLATURE}

$\mathrm{B}$ blowing on

$\mathrm{C}_{1}$ rolling moment coefficient

$\mathrm{C}_{\beta} \quad \partial \mathrm{C}_{\mathrm{l}} / \partial \beta, \mathrm{deg}^{-1}$

Aerospace Engineer, Senior Member AIAA

${ }^{\dagger}$ Aerospace Engineer, Member AIAA

" Research Associate, Student Member AIAA

Graduate Student

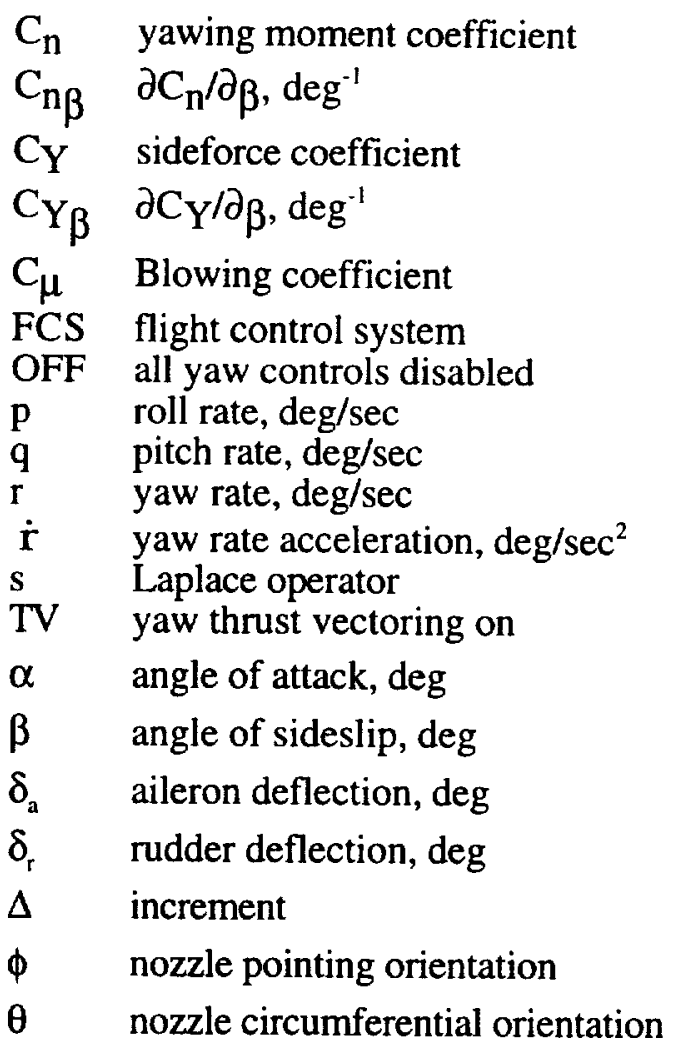

\section{INTRODUCTION}

Emphasis in expansion of the conventional flight envelope of fighter aircraft has 
stimulated research in innovative concepts for high-angle-of-attack control. At high angles of attack, the need for large yawing moments for roll coordination is in conflict with conventional tail effectiveness which decreases as the tail is immersed in the low energy wake of the wing. Two of the primary concepts which manipulate the forebody flow to provide yawing moments that have been studied are mechanical systems (deflectable forebody strakes) and pneumatic systems (forebody blowing). Work with the mechanical systems has progressed from initial concept development ${ }^{1}$ to flight demonstration on the NASA HighAngle-of-Attack Research Vehicle (HARV). Pneumatic forebody controls have been investigated through numerous static wind tunnel and analytical studies ${ }^{2-7}$. A flight demonstration of control power available with forebody blowing was conducted with the X-29 aircraft using a system where the pilot manually opened and closed valves to control blowing on the forebody ${ }^{8}$. The X-29 experiment demonstrated the validity of the wind tunnel derived moments obtained with forebody blowing. A simulation study evaluating on/off forebody blowing integrated into a flight control system ${ }^{9}$ for high angle-of-attack control showed that the concept was promising.

This paper highlights results of the first flight demonstration of forebody blowing for yaw control at high angles of attack integrated into a flight control system for stability augmentation as well as control. A primary objective of the test was to evaluate the feasibility of pneumatic controls with an on/off control scheme in light of questions such as whether the lags associated with pneumatic controls would inhibit the effectiveness as a closed loop controller. A generic fighter airplane configuration was selected which was fitted with conventional, thrust vectoring, and forebody pneumatic controls. A flight control system was developed, and the effectiveness of pneumatic controls was demonstrated in flight without the use of rudders or thrust vectoring for additional yaw control. Data obtained in a previous static wind tunnel test were also compared with the dynamic test results.

\section{DESCRIPTION OF TEST}

\section{Model}

The model used in this study was a generic fighter model with a circular cross-section fuselage, flat-plate wing and tail surfaces with sharp double-beveled leading and trailing edges (fig 1). A similar model for captive wind tunnel tests has been extensively tested $^{10,11}$. The model used in the current study was built to allow free-flight testing of configurational effects and forebody controls. The roll/yaw inertia ratio $(\mathrm{Ix} / \mathrm{lz}=7.74)$, which is a dominant factor in the lateraldirectional dynamic response, was representative of many current fighter aircraft.
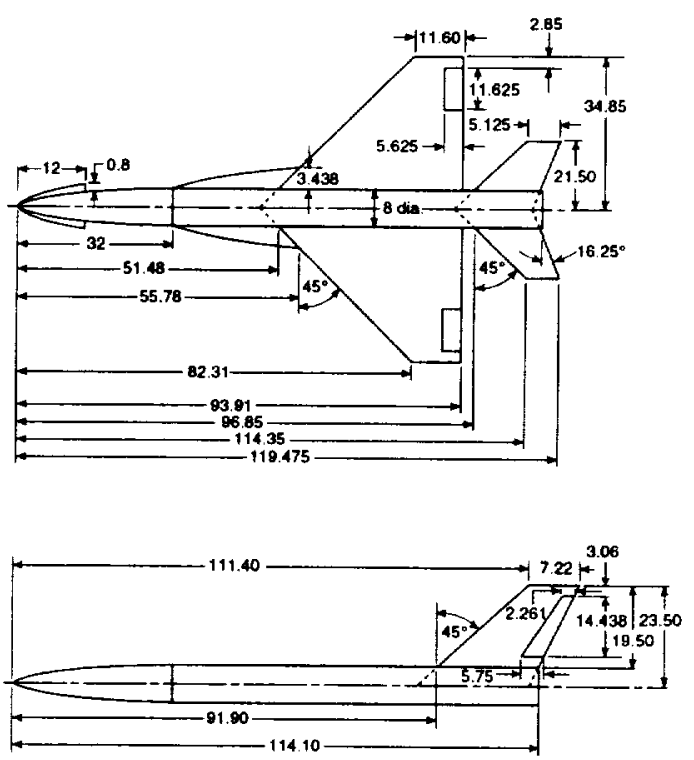

Figure 1. Sketch of model. (dimensions in inches)

The forebody of the model was a circular cross-section tangent ogive with a fineness ratio of 4 with strakes protruding normal to the surface at the $75^{\circ}$ radial location from the top of the forebody as shown in figure 1 .

Slotted blowing nozzles oriented as shown in figure 2 were also incorporated on the forebody. High pressure air was supplied to the nozzles through valves which were operated by servos controlled by the flight 
control computer. Calibrations of mass flow rate and supplied pressure were conducted prior to the flight test series, and pressures were selected based on tunnel speed to obtain the desired $\mathrm{C} \mu$. The pneumatic lag of the system beyond the valves was not measured; however, the valves were connected to the nozzles through 0.213 "I.D. tubing over a 12 " distance. The valve characteristics were such that the mass flow rate through the valve was essentially 0 until the valve reached $30 \%$ open. At $60 \%$ open, the mass flow rate was at nearly the maximum value which remained constant as the valve opened further. All free-flight tests were conducted at $C_{\mu} \approx$ 0.0077 .

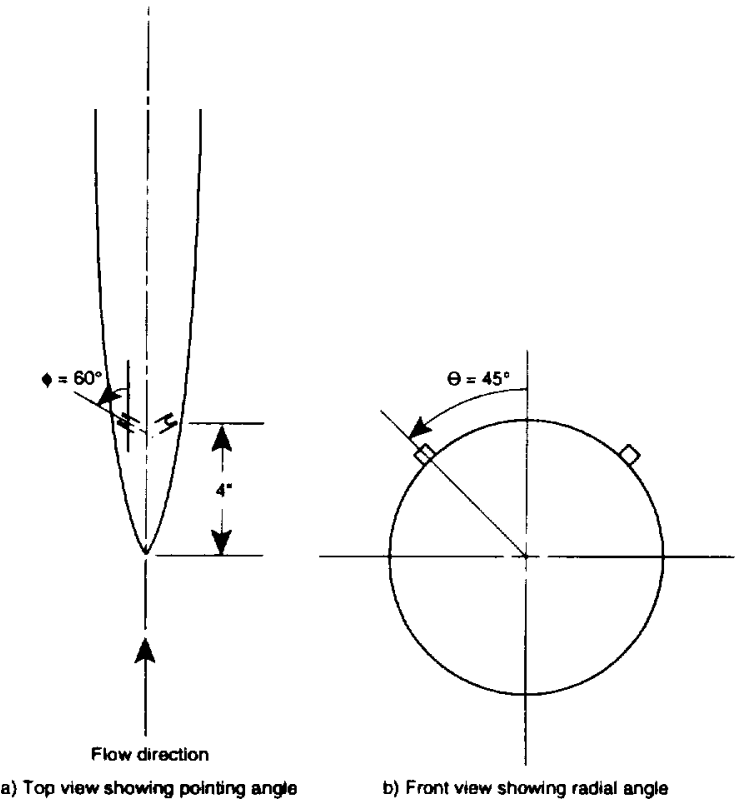

Figure 2. Forebody nozzle orientation.

In addition to forebody blowing, the model incorporated ailerons, rudders, differential horizontal tail deflections and yaw thrust vectoring for lateral-directional control, and symmetric horizontal tail deflections and pitch thrust vectoring for pitch control.

\section{Free-Flight Test Technique}

The wind tunnel free-flight tests were conducted in the Langley 30 - by 60 -Foot Tunnel with the technique illustrated in figure 3. With this technique, the remotely controlled dynamically scaled model was flown in the open test section of the 30 - by 60 -Foot Tunnel. A photograph of the model flying during the test is shown in figure 4. The wind tunnel free-flight tests were used to evaluate first the flying characteristics of the model with the various control laws, and then to evaluate controllability with forebody blowing as the only yaw controller. Model motions were measured and pilot comments recorded for each flight condition.

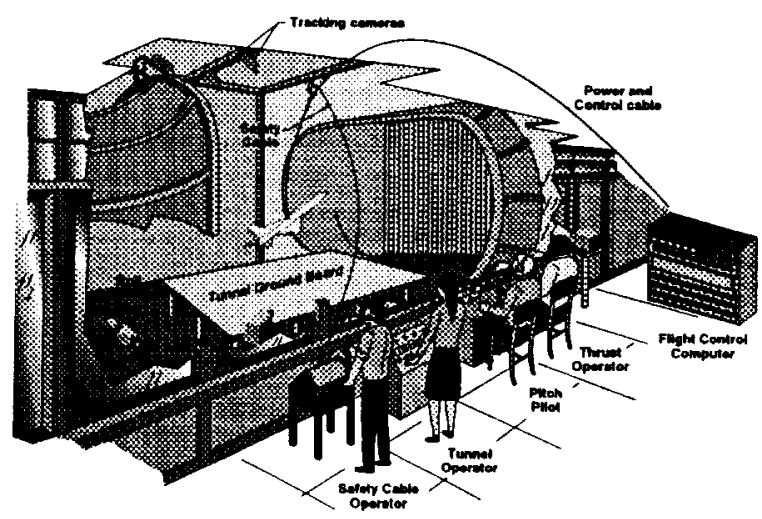

Figure 3. Free-flight test technique.

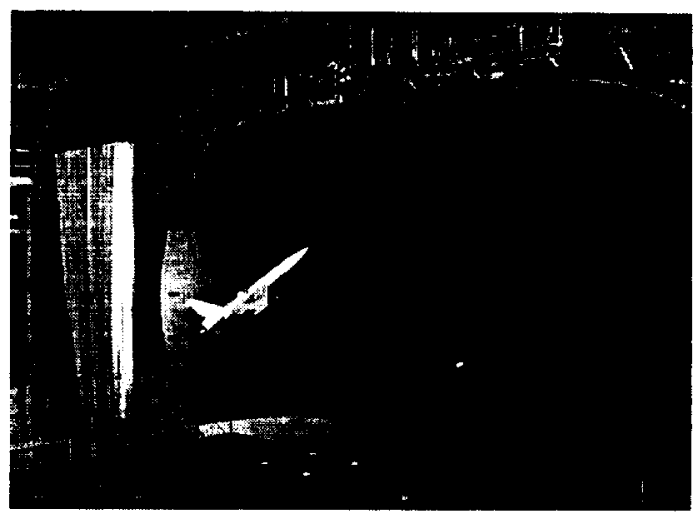

Figure 4. Generic model during free-flight.

During the free-flight tests, the model was attached to an umbilical chord which supplied pneumatic and electrical power and control signals to the model. The chord also contained a $1 / 8$ " steel safety cable that was controlled by a safety cable operator using a high-speed pneumatic winch. The safety cable operator helped launch the model at the start of a test, retrieve the model at the end of a test, keep tension off the model from the umbilical cable during the test, and would 
attempt to protect the model in an out-ofcontrol situation by pulling the model out of the airstream.

In addition to the safety cable operator, the model flight crew consisted of a pitch pilot, thrust pilot, and a roll/yaw pilot. These piloting functions were located in the positions shown in figure 3 to afford the best view for controlling the pertinent axes. The separation of the piloting duties is very advantageous for several reasons. By separating pilots by axes, effective evaluations can more easily be obtained since the pilot controls only the axes he evaluates. Control of the model is also enhanced by providing the optimal visual perspective for control of each axis. Due to dynamic scaling, the model motions are substantially faster than that of a full-scale airplane, so separation of piloting tasks is beneficial for that reason as well.

The primary component in the free-flight control system (FCS) is a digital minicomputer programmed with the flight control laws. The computer processed sensor information from the model and command inputs from the pilots to generate command signals that drive the high-speed pneumatic actuators onboard the model. The data sensors on the model included a threeaxis rate gyro to measure angular rates, an accelerometer package to measure normal, axial and side-force accelerations, a boommounted $\alpha / \beta$ vane sensor on the starboard wing tip for angle of attack and sideslip, potentiometers to measure control surface positions, and a transducer to measure pressure at forebody valves for mass flow calculations. These sensor data, along with pilot control inputs, were recorded in the computer for post-flight analysis. Angular rates, linear accelerations and $\alpha / \beta$ vane sensor data were filtered with a first order lag filter with a cut-off frequency of $20 \mathrm{Hertz}$ before entering the FCS. Additionally, $\alpha$ and $\beta$ from the wingtip mounted vanes were corrected for angular rates, upwash, and sidewash. Post-flight data reduction included calculation of angular accelerations by differentiation of measured angular rate data.

\section{Control System Description}

A flight control system was designed using the measured static aerodynamic characteristics. The control system utilized gains scheduled with angle of attack for state feedbacks (p,q,r) and for static stability augmentation. The design of the flight control system was evaluated using linearized aerodynamic math models over the range of angle of attack expected to be flown. The complete control laws were then inplemented on the flight control computer for the freeflight tests. During the tests, severe electrical noise problems on several channels to and from the model occurred, and due to time constraints, those channels were deactivated. Loss of those channels resulted in fixed stabilator and rudder positions, with only pitch and yaw thrust vectoring, ailerons, and forebody blowing available for control. Control system gains were not modified to account for the deactivation of the rudder and horizontal tails. All of the data presented herein was flown with the stabilators and rudder at a fixed deflection of $0^{\circ}$. The flight control system block diagram (without rudder and stabilators) is shown in figure 5 .

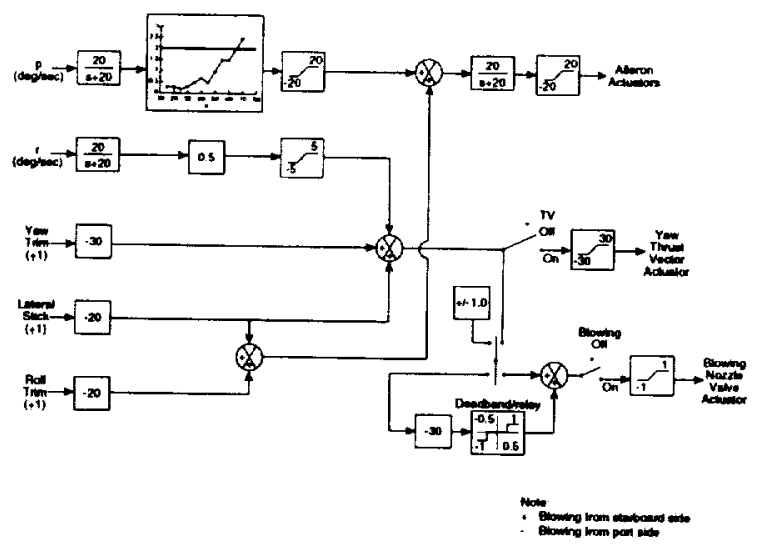

Figure 5. Flight control system block diagram.

\section{AERODYNAMIC CHARACTERISTICS}

\section{Static Results}

Static wind tunnel tests were conducted with the model in the Langley 30 - by 60 - Foot 
Tunnel to evaluate the effectiveness of the slotted nozzles. The basic stability and control characteristics of the configuration were obtained in addition to the effectiveness of the blowing concept. A summary of the data will be presented here. Figure 6 shows the static lateral-directional stability characteristics of the configuration.

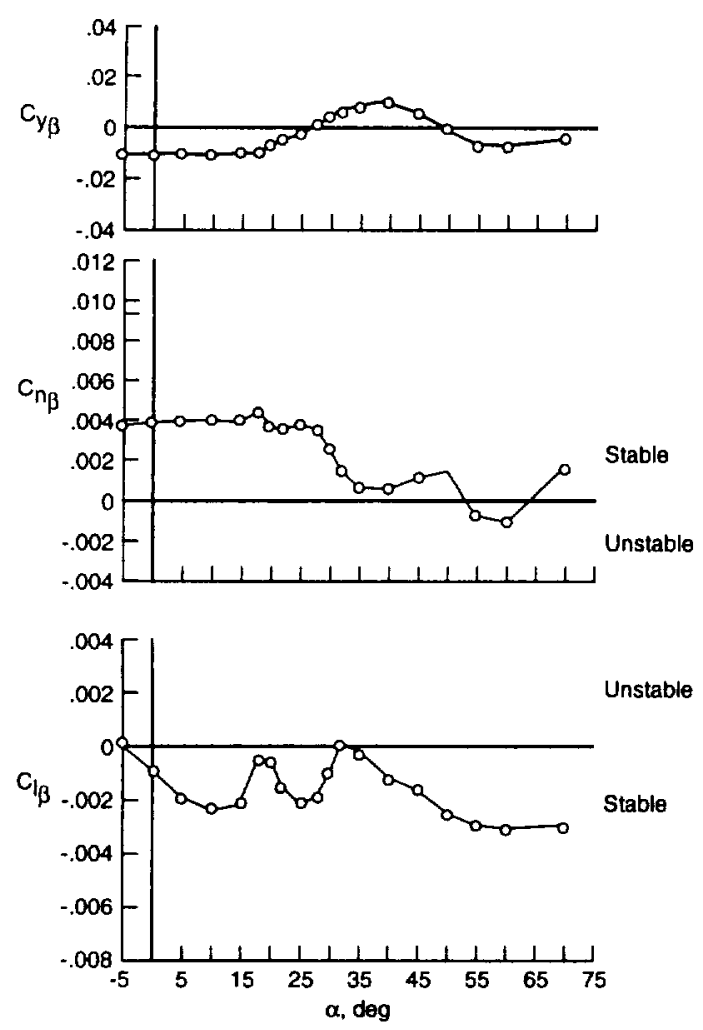

Figure 6. Unaugmented static lateraldirectional stability.

At angles of attack below $25^{\circ}$, the configuration exhibits static directional stability. Above $\alpha=25^{\circ}$, the directional stability rapidly decreases to neutral stability by approximately $\alpha=35^{\circ}$. Corresponding lateral stability data also show unstable dihedral effect at $\alpha=35^{\circ}$. These characteristics would predict yaw departure susceptibility in this region.

Control power available is shown in figure 7 for the conventional aerodynamic control effectors. These data show that the rudder control power decreases rapidly at $\alpha>30^{\circ}$.
This is typical of most airplane designs; however, as flight is conducted at higher angles of attack, more yaw control is required to coordinate turns. This is the primary reason alternate control devices, such as thrust vectoring and forebody vortex controls, have been studied. The data also show a slight adverse yaw due to aileron deflection above $\alpha=20^{\circ}$. Aileron control power also diminishes rapidly as the wing stall progresses.

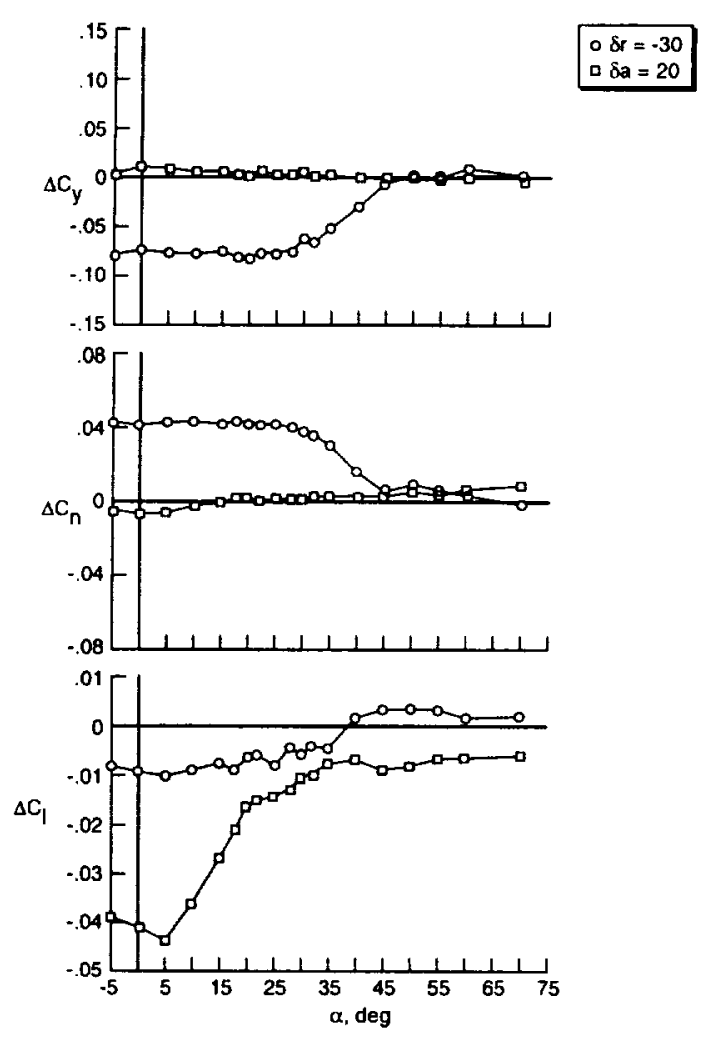

Figure 7. Effectiveness of conventional controls.

Figure 8 shows the effectiveness of blowing on the lateral-directional coefficients. The results indicate that the moments produced are nonlinear with mass flow rate; however, the nozzles do generate large amounts of yawing moment control over a large angle-ofattack range. Additionally, a large rolling moment in the opposite direction of yaw is developed near the stall angle of attack of $35^{\circ}$. This result is different than seen in previous studies of other configurations ${ }^{4.5}$ where forebody blowing produced yawing and 
rolling moments in the same direction. Based on these results, and on the physics involved, rolling moments produced by forebody vortex control are expected to be highly configuration dependent.

\section{Free-Flight Results}

As mentioned previously, all free-flight data were obtained without the use of rudder or horizontal tail movements, and for all of the flight data discussed, those surfaces were set to $0^{\circ}$. Flight tests were conducted with the forebody blowing system in both open and closed loop fashions. The open loop tests were conducted by stabilizing the model with conventional and thrust vectoring controls, and then short step inputs from a nozzle were made. The resultant model motions were then analyzed to calculate the effectiveness of the nozzle at that flight condition and comparisons were made to static wind tunnel predictions. Figure 9 shows the generally favorable comparison with the starboard nozzle effectiveness obtained in flight with that measured during static wind tunnel tests. The data show slightly more yaw control available during flight at the higher angles of attack than was predicted in the static tests.

Further, the time lag between the nozzle opening and model motion gave an indication of the flow lags associated with this controller. An example is shown in figure 10 which indicates a time lag of approximately 0.2 seconds, model scale, between the point at which the valve opens beyond $30 \%$, and the resulting maximum moment (yaw acceleration) on the model. Implementation of a pneumatic system on a full-scale airplane should result in smaller lags if the system is designed with fast acting valves very close to the nozzles on the forebody. Nevertheless, even with the large lags seen in the model setup, the system produced useable control moments for adequate flying qualities during the free-flight test.

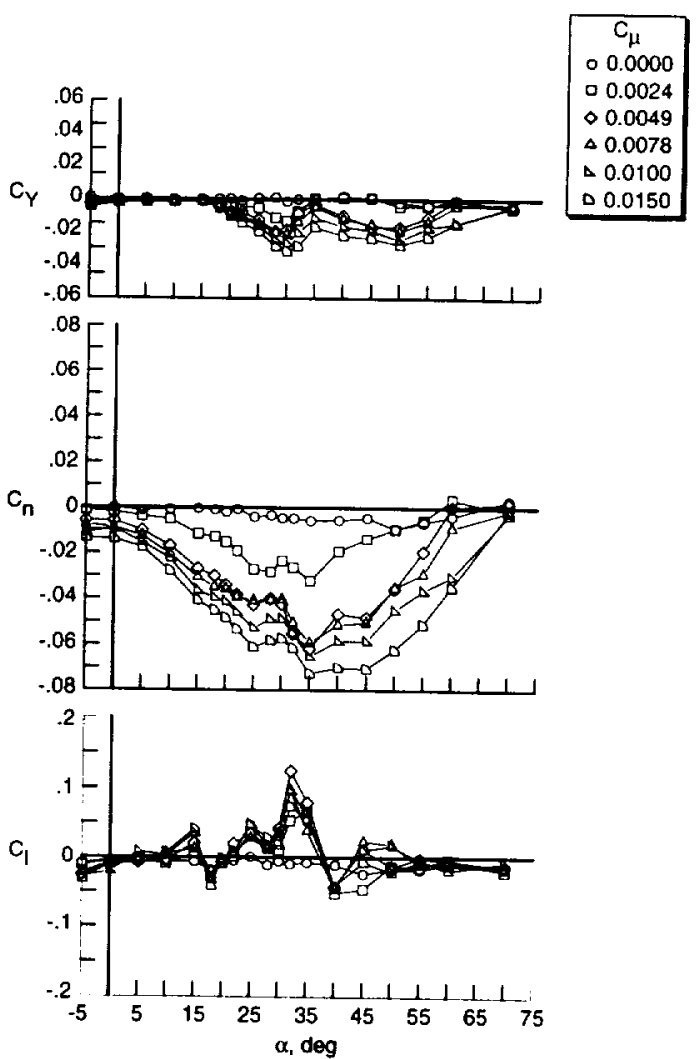

Figure 8. Effectiveness of forebody blowing. Starboard nozzle.

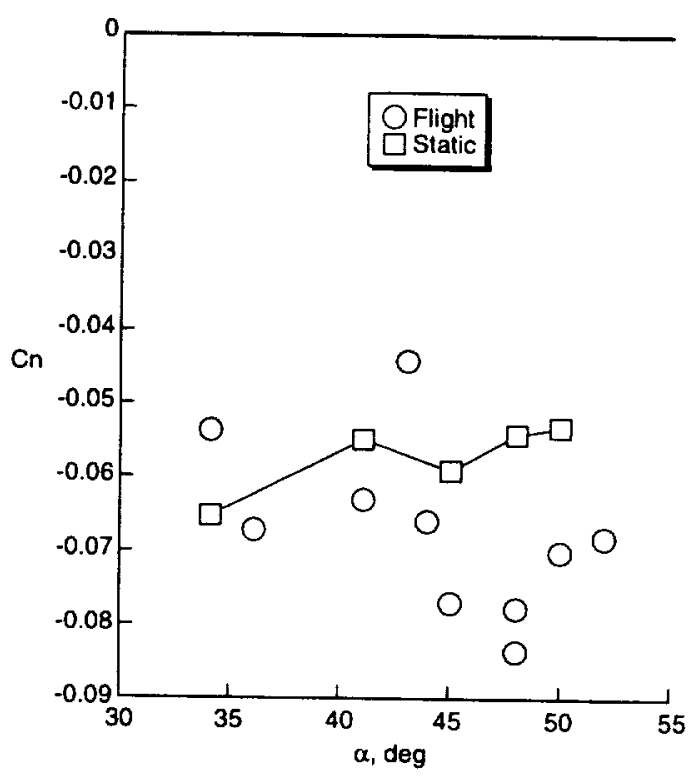

Figure 9. Comparison of static and flight derived forebody blowing effectiveness. 


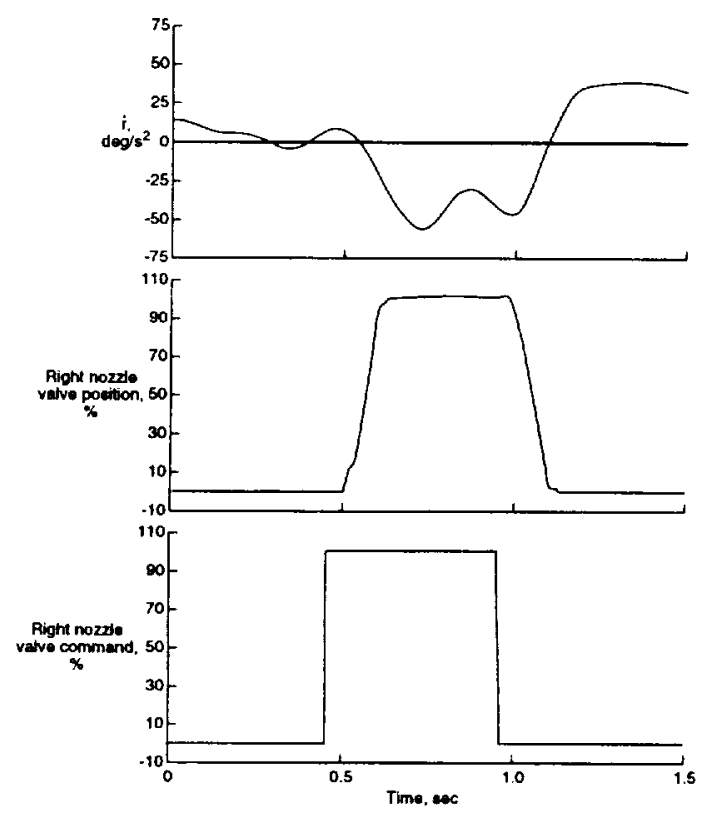

Figure 10. Pneumatic lag.

Closed loop evaluations were made by flying initially with thrust vectoring only for pitch and yaw. After confidence was gained with the model controllability, forebody blowing was added to the controls.
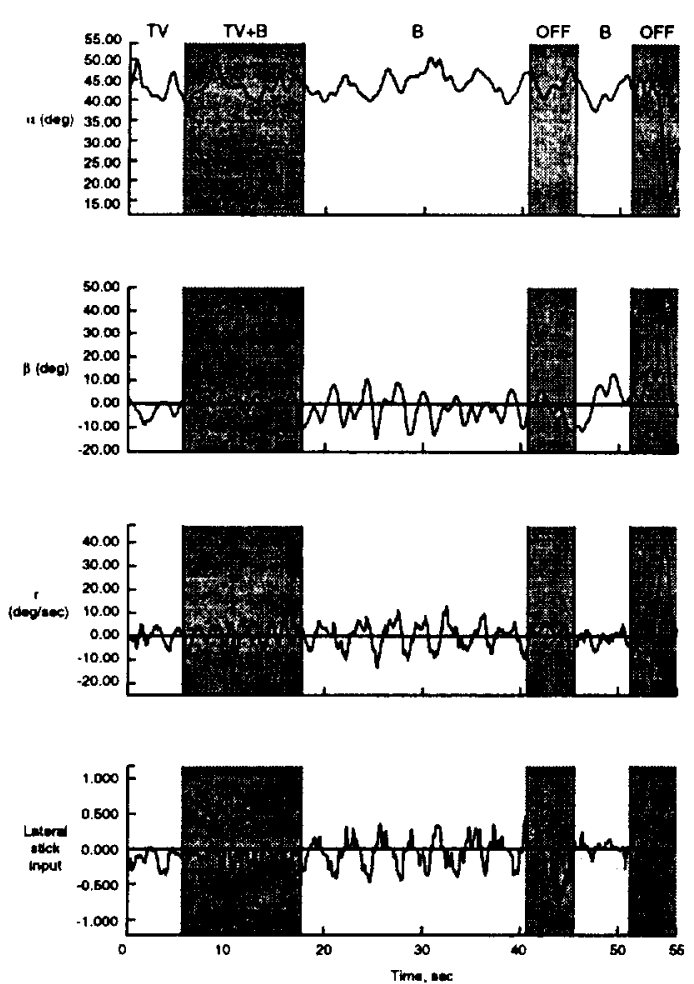

Figure 11. Typical time history of free-flight demonstration.
This was followed by the disabling of the yaw vectoring so that total yaw control was obtained by the forebody blowing. Finally, forebody blowing was turned off, which resulted in the departure of the model. A time history plot of one flight sequence is shown in figure 11 .

During flight sequences such as shown in figure 11, pilot comments indicated slightly better controllability when blowing was added to the yaw thrust vectoring control. When the thrust vectoring was turned off, a noticeable increase in pilot workload, sideslip excursions and model motion were noted. Pilot comments indicated that the model was still very controllable; however, yaw control was "looser" and less predictable resulting in larger model excursions. It should be noted that the flight control system gains were originally designed for use with rudder and blowing in combination. When electrical problems resulted in the disabling of the rudder, the gains for the forebody blowing were not changed to reflect this, so they were not optimized in any way. Refinements of the flight control system gains would probably result in better flying qualities.

The last three sequences in the data of figure 11 show what happened when the blowing was turned off. In this condition, pitch thrust vectoring and ailerons were the only active control devices. The model started flying out of the test section area and could not be recovered with full lateral stick control, so the blowing was turned back on and the model quickly recovered. The blowing was again turned off, and the model exhibited a nose slice departure.

One aspect which is important for the design of a successful system in flight, is the capability to generate sufficient mass flow to provide adequate control. With the tested control scheme, figure 12 shows the percentage of time a forebody control valve was open during the flights with forebody controls used as the sole yaw control device. 


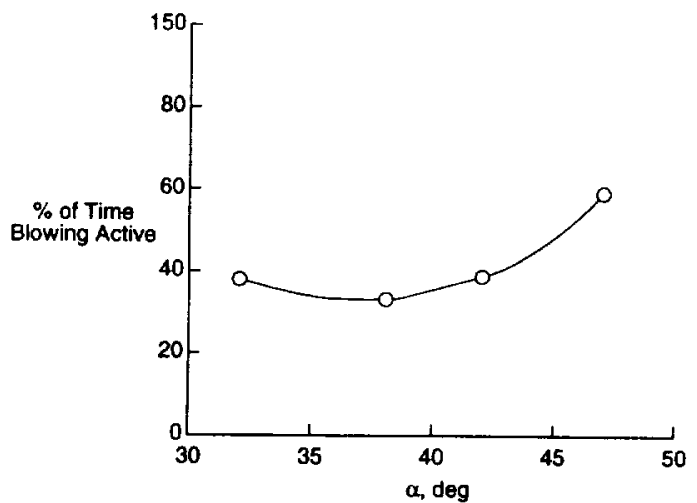

Figure 12. Blowing utilization.

\section{CONCLUSIONS}

A model equipped with forebody pneumatic vortex controls was flown in the NASA Langley 30 - by 60 -Foot Tunnel. This represents the first flight demonstration of forebody pneumatic controls integrated in a closed-loop flight control system. Results of the test indicated good correlation with static predictions, and also showed the on/off control scheme produced acceptable flying qualities and stability augmentation for this test. The model was successfully flown without rudder or thrust vectoring controls for yaw to beyond $45^{\circ}$ angle of attack. During flight, the nozzles were active approximately $40-60 \%$ of the time, which indicates the blowing capability requirements for such a system.

\section{REFERENCES}

1. Rao, D. M.; and Murri, D. G.:

Exploratory Investigation of Deflectable Forebody Strakes for High Angle-ofAttack Yaw Control. AIAA-86-0333, January 1986.

2. Skow, A. M.; Moore, W. A.; and Lorincz, D. J.: Forebody Vortex Blowing - A Novel Control Concept to Enhance Departure/Spin Recovery Characteristics of Fighter and Trainer Aircraft. AGARD CP-262, Paper No. 24, 1979.

3. Mosbarger, N. A.: Vortex Control with Jet Blowing on a F-16 Forebody. AIAA94-1830-CP.

4. O'Rourke, M. J.: Experimental Investigation of Slot Blowing for Yaw Control on a Generic Fighter
Configuration With a Chined Forebody. AIAA-95-1798, June 1995.

5. O'Rourke, M. J.; and Sedor, J. L.: Forebody-Mounted Jet Nozzles For Yaw Control on a Generic Fighter Configuration With a Chined Forebody. AIAA-95-3490, August, 1995.

6. Tavella, D. A.; Schiff, L. B. ; and Cummings, R. M.: Pneumatic Vortical Flow Control at High Angles of Attack. AIAA-90-0098, January, 1990.

7. Gee, K.: Computational Analysis of Forebody Tangential Slot Blowing on the High Alpha Research Vehicle - Final Report. NASA-CR-197754, 1995.

8. Smith, W.: X-29 High AOA Flight Test Results: An Overview. SAE-931367, April 1993.

9. Adams, R. J.; and Buffington, J. M.: Design and Analysis of Modification For VISTA F-16 High Angle-of-Attack Envelope Expansion. WL-TR-93-3064, July 1993.

10. Nguyen; Whipple; and Brandon: Recent Experiences of Unsteady Aerodynamic Effects on Aircraft Flight Dynamics at High Angle of Attack. AGARD-CP-386 May 1985.

11. Brandon, J.M.; Murri, D.G.; and Nguyen, L.T.: Experimental Study of Effects of Forebody Geometry on High Angle of Attack Static and Dynamic Stability and Control. ICAS-86-5.4.1, September 1986. 
